\title{
Related Fixed Point of Set-Valued Mappings in Three Menger Spaces
}

\author{
Ismat Beg ${ }^{1}$ and Sunny Chauhan ${ }^{2}$ \\ ${ }^{1}$ Centre for Applicable Mathematics and Statistics, University of Central Punjab, Lahore 54770, Pakistan \\ ${ }^{2}$ R. H. Government Postgraduate College, Kashipur, Udham Singh Nagar, Uttarakhand 244 713, India \\ Correspondence should be addressed to Ismat Beg; ibeg@lums.edu.pk
}

Received 22 November 2012; Revised 20 February 2013; Accepted 3 March 2013

Academic Editor: Alexandre Timonov

Copyright (c) 2013 I. Beg and S. Chauhan. This is an open access article distributed under the Creative Commons Attribution License, which permits unrestricted use, distribution, and reproduction in any medium, provided the original work is properly cited.

We prove a related fixed point theorem for set-valued mappings in three Menger spaces. Some examples are also furnished to support the results. Our main result generalizes and extends several known results in the literature.

\section{Introduction}

Fisher [1] initiated the study of conditions for the existence of a relation connecting the fixed points of two mappings in two different metric spaces (also see [2]). Afterward, Fisher and Türkoğlu [3] proved a related fixed point theorem for setvalued mappings in two metric spaces. In 2003, Chourasia and Fisher [4] established a related fixed point theorem for two pairs of set valued mappings in two metric spaces. Several mathematicians have extensively developed metrical related fixed point theorems (see, e.g., [5-8]). Later on, Pant [9] firstly studied related fixed point theorems for single valued mappings in two complete Menger spaces and generalized the results of Fisher $[1,2]$. Pant and Kumar [10] extended the results of Namdeo et al. [7] to two pairs of single-valued mappings.

In this paper, we prove a related fixed point theorem for set-valued mappings in three complete Menger spaces. Our result generalizes the result of Jain et al. [6] and extends the result of Fisher and Türkoğlu [3].

\section{Preliminaries}

Definition 1 (see [11]). A mapping $\Delta:[0,1] \times[0,1] \rightarrow[0,1]$ is called a $t$-norm if

(i) $\Delta(a, 1)=a, \Delta(0,0)=0$,

(ii) $\Delta(a, b)=\Delta(b, a)$, (iii) $\Delta(a, b) \leq \Delta(c, d)$, whenever $a \leq c$ and $b \leq d$,

(iv) $\Delta(a, \Delta(b, c))=\Delta(\Delta(a, b), c)$, for all $a, b, c, d \in[0,1]$.

Examples of $t$-norms are $\Delta_{M}(a, b)=\min \{a, b\}, \Delta_{P}$ $(a, b)=a b$, and $\Delta_{L}(a, b)=\max \{a+b-1,0\}$.

Definition 2 (see [11]). A mapping $F: \mathbb{R} \rightarrow \mathbb{R}^{+}$is called distribution function if it is nondecreasing, left continuous with $\inf \{F(t): t \in \mathbb{R}\}=0$ and $\sup \{F(t): t \in \mathbb{R}\}=1$.

Let $\mathfrak{I}$ be the set of all distribution functions whereas $H$ stands for the specific distribution function (also known as Heaviside function) defined by

$$
H(t)= \begin{cases}0, & \text { if } t \leq 0 \\ 1, & \text { if } t>0\end{cases}
$$

Definition 3 (see [11]). Let $X$ be a nonempty set. An ordered pair $(X, \mathscr{F})$ is called a PM-space where $\mathscr{F}$ is a mapping from $\mathscr{F}: X \times X \rightarrow \mathfrak{J}$ satisfying the following conditions:

(i) $F_{x, y}(t)=H(t)$ if and only if $x=y$,

(ii) $F_{x, y}(t)=F_{y, x}(t)$,

(iii) $F_{x, z}(t)=1$ and $F_{z, y}(s)=1$; then $F_{x, y}(t+s)=1$ for all $x, y, z \in X$ and $t, s>0$.

Every metric space $(X, d)$ can be realized as a PMspace by considering $\mathscr{F}: X \times X \rightarrow \mathfrak{I}$ defined by 
$F_{x, y}(t)=H(t-d(x, y))$ for all $x, y \in X$. So PM-spaces offer a wider framework (than that of the metric spaces) and are general enough to cover even wider statistical situations.

Definition 4 (see [12]). A Menger space $(X, \mathscr{F}, \Delta)$ is a triplet where $(X, \mathscr{F})$ is a PM-space and $\Delta$ is a $t$-norm satisfying the following condition:

$$
F_{x, y}(t+s) \geq \Delta\left(F_{x, z}(t), F_{z, y}(s)\right)
$$

for all $x, y, z \in X$ and $t, s>0$.

Definition 5 (see [11]). Let $(X, \mathscr{F}, \Delta)$ be a Menger space and $\Delta$ a continuous $t$-norm. A sequence $\left\{x_{n}\right\}$ in $X$ is said to be

(i) convergent to a point $x$ in $X$ if and only if for every $\varepsilon>0$ and $\lambda>0$, there exists a positive integer $N(\varepsilon, \lambda)$ such that $F_{x_{n}, x}(\varepsilon)>1-\lambda$ for all $n \geq N(\varepsilon, \lambda)$.

(ii) Cauchy if for every $\varepsilon>0$ and $\lambda \in(0,1)$, there exists a positive integer $N(\varepsilon, \lambda)$ such that $F_{x_{n}, x_{m}}(\varepsilon)>1-\lambda$ for all $n, m \geq N(\varepsilon, \lambda)$.

A Menger space in which every Cauchy sequence is convergent is said to be complete.

Lemma 6 (see $[13])$. Let $(X, \mathscr{F}, \Delta)$ be a Menger space. If there exists a constant $k \in(0,1)$ such that

$$
F_{x, y}(k t) \geq F_{x, y}(t)
$$

for all $t>0$ with fixed $x, y \in X$, then $x=y$.

We need the following definition due to Chang et al. [14] for subsequent use in next section.

Definition 7 (see [14]). Let $(X, \mathscr{F}, \Delta)$ be a Menger space and $\mathcal{U}$ a nonempty subset of $X$. Then $\mathcal{U}$ is said to be probabilistically bounded if $\sup _{t>0} \inf _{x, y \in \mathcal{U}} F_{x, y}(t)=1$.

If $X$ is itself probabilistically bounded, then $X$ is said to be a probabilistically bounded space.

Let $\mathscr{C} \mathscr{B}(X)$ be a family of nonempty closed and bounded subsets of a Menger space $(X, \mathscr{F}, \Delta)$. For all $\mathcal{U}, \mathscr{V} \in \mathscr{C} \mathscr{B}(X)$ and for every $t>0$, we define

$$
\begin{aligned}
& { }_{D} F_{\mathscr{U}, \mathscr{V}}(t)=\sup \left\{F_{a, b}(t) ; a \in \mathcal{U}, b \in \mathscr{V}\right\}, \\
& { }_{\delta} F_{\mathscr{U}, \mathscr{V}}(t)=\inf \left\{F_{a, b}(t) ; a \in \mathscr{U}, b \in \mathscr{V}\right\} .
\end{aligned}
$$

If the set $\mathcal{U}$ consists of a single point $a$, we write ${ }_{\delta} F_{\mathscr{U}, \mathscr{V}}(t)=$ ${ }_{\delta} F_{a, \mathscr{V}}(t)$. Also if the set $\mathscr{V}$ consists of a single point $b$, we write ${ }_{\delta} F_{\mathscr{U}, \mathscr{V}}(t)={ }_{\delta} F_{a, b}(t)$. It follows immediately from the definition that

(i) ${ }_{\delta} F_{\mathscr{U}, \mathscr{V}}(t)={ }_{\delta} F_{\mathscr{V}, \mathscr{U}}(t) \geq 0$,

(ii) ${ }_{\delta} F_{\mathscr{U}, \mathscr{V}}(t)=1$ if and only if $\mathscr{U}=\mathscr{V}=\{a\}$, for all $\mathscr{U}, \mathscr{V} \in \mathscr{C} \mathscr{B}(X)$.

\section{Main Result}

Now we prove a related fixed point theorem for set-valued mappings in three complete Menger spaces.
Theorem 8. Let $(X, \mathscr{F}, \Delta),(Y, \mathscr{G}, \Delta)$, and $(Z, H, \Delta)$ be three complete Menger spaces, where $\Delta$ is a continuous $t$-norm. If $P$ is a continuous mapping of $X$ into $\mathscr{C} \mathscr{B}(Y), Q$ is a continuous mapping of $Y$ into $\mathscr{C} \mathscr{B}(Z)$, and $R$ is a mapping of $Z$ into $\mathscr{C} \mathscr{B}(X)$ satisfying the inequalities

$$
\begin{gathered}
{ }_{\delta} F_{R Q P x, R Q P x^{\prime}}(k t) \geq \min \left\{F_{x, x^{\prime}}(t),{ }_{\delta} F_{x, R Q P x}(t),\right. \\
{ }_{\delta} F_{x^{\prime}, R Q P x^{\prime}}(t),{ }_{\delta} G_{P x, P x^{\prime}}(t), \\
\left.{ }_{\delta} H_{\mathrm{QP} x, \mathrm{Q} P x^{\prime}}(t)\right\}, \\
{ }_{\delta} G_{P R Q y, P R Q y^{\prime}}(k t) \geq \min \left\{G_{y, y^{\prime}}(t),{ }_{\delta} G_{y, P R Q y}(t),\right. \\
{ }_{\delta} G_{y^{\prime}, P R Q y^{\prime}}(t),{ }_{\delta} H_{\mathrm{Q} y, \mathrm{Q} y^{\prime}}(t), \\
{ }_{\delta} F_{R Q} H_{\mathrm{QPR} z, \mathrm{Q} P R z^{\prime}}(k t) \geq \min \left\{H_{z, z^{\prime}}(t),{ }_{\delta} H_{z, \mathrm{Q} P R z}(t)\right\}, \\
{ }_{\delta} H_{z^{\prime}, \mathrm{QPR} z^{\prime}}(t),{ }_{\delta} F_{R z, R z^{\prime}}(t), \\
\left.{ }_{\delta} G_{P R z, P R z^{\prime}}(t)\right\}
\end{gathered}
$$

for all $x, x^{\prime} \in X, y, y^{\prime} \in Y, z, z^{\prime} \in Z, k \in(0,1)$ and $t>0$, Then $R Q P$ has a unique fixed point $u$ in $X, P R Q$ has a unique fixed point $v$ in $Y$, and $Q P R$ has a unique fixed point $w$ in $Z$. Further $P u=\{v\}, Q v=\{w\}$, and $R w=\{u\}$.

Proof. Let $x=x_{1}$ be an arbitrary point in $X$. We define the sequences $\left\{x_{n}\right\}$ in $X,\left\{y_{n}\right\}$ in $Y$, and $\left\{z_{n}\right\}$ in $Z$ inductively as follows. We choose a point $y_{1}$ in $P x_{1}$, then a point $z_{1}$ in $Q y_{1}$, and then a point $x_{2}$ in $R z_{1}$. In general, having chosen $x_{n}$ in $X$, $y_{n}$ in $Y$, and $z_{n}$ in $Z$, choose a point $x_{n+1}$ in $R z_{n}$, then a point $y_{n+1}$ in $P x_{n+1}$, and then a point $z_{n+1}$ in $Q y_{n+1}$ for $n=1,2, \ldots$. Applying inequality (5), we get

$$
\begin{aligned}
& F_{x_{n+1}, x_{n+2}}(k t) \geq{ }_{\delta} F_{R Q P x_{n}, R Q P x_{n+1}}(k t) \\
& \geq \min \left\{F_{x_{n}, x_{n+1}}(t),{ }_{\delta} F_{x_{n}, R Q P x_{n}}(t)\right. \text {, } \\
& { }_{\delta} F_{x_{n+1}, R Q P x_{n+1}}(t),{ }_{\delta} G_{P x_{n}, P x_{n+1}}(t) \text {, } \\
& \left.{ }_{\delta} H_{\mathrm{QPx} x_{n}, \mathrm{QP} x_{n+1}}(t)\right\} \text {, } \\
& \geq \min \left\{{ }_{\delta} F_{R Q P x_{n-1}, R Q P x_{n}}(t)\right. \text {, } \\
& { }_{\delta} F_{R Q P x_{n}, R Q P x_{n+1}}(t) \text {, } \\
& { }_{\delta} G_{P R Q y_{n-1}, P R Q y_{n}}(t), \\
& \left.{ }_{\delta} H_{\mathrm{QPR} z_{n-1}, \mathrm{QPR} z_{n}}(t)\right\}, \\
& \geq \min \left\{{ }_{\delta} F_{R Q P x_{n-1}, R Q P x_{n}}(t)\right. \text {, } \\
& { }_{\delta} G_{P R Q y_{n-1}, P R Q y_{n}}(t) \text {, } \\
& \left.{ }_{\delta} H_{\mathrm{QPR} z_{n-1}, \mathrm{QPR} z_{n}}(t)\right\} \text {. }
\end{aligned}
$$


Using inequality (6), we get

$$
\begin{aligned}
& G_{y_{n+1}, y_{n+2}}(k t) \geq{ }_{\delta} G_{P R Q y_{n}, P R Q y_{n+1}}(k t) \\
& \geq \min \left\{G_{y_{n}, y_{n+1}}(t),{ }_{\delta} G_{y_{n}, P R Q y_{n}}(t)\right. \text {, } \\
& { }_{\delta} G_{y_{n+1}, P R Q y_{n+1}}(t),{ }_{\delta} H_{\mathrm{Q} y_{n}, \mathrm{Q} y_{n+1}}(t), \\
& \left.{ }_{\delta} F_{R Q y_{n}, R Q y_{n+1}}(t)\right\},
\end{aligned}
$$

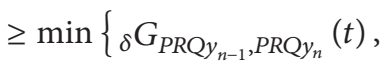

$$
\begin{aligned}
& { }_{\delta} G_{P R Q y_{n}, P R Q y_{n+1}}(t), \\
& { }_{\delta} H_{\mathrm{QPR} z_{n-1}, \mathrm{QPR} z_{n}}(t) \text {, } \\
& \left.{ }_{\delta} F_{R Q P x_{n}, R Q P x_{n+1}}(t)\right\},
\end{aligned}
$$

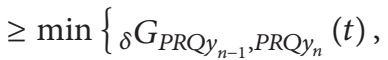

$$
\begin{aligned}
& { }_{\delta} H_{\mathrm{QPR} z_{n-1}, \mathrm{QPR} z_{n}}(t), \\
& \left.{ }_{\delta} F_{R Q P x_{n}, R Q P x_{n+1}}(t)\right\},
\end{aligned}
$$

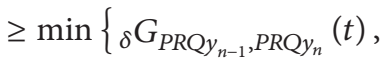

$$
\begin{aligned}
& { }_{\delta} H_{\mathrm{QPRz} z_{n-1}, \mathrm{QPRz} z_{n}}(t) \text {, } \\
& \left.{ }_{\delta} F_{R Q P x_{n-1}, R Q P x_{n}}(t)\right\} \text {, }
\end{aligned}
$$

on using inequality (8). Further, on using inequality (7), we have

$$
\begin{aligned}
& H_{z_{n+1}, z_{n+2}}(k t) \geq{ }_{\delta} H_{\mathrm{QPR} z_{n}, \mathrm{QPR} z_{n+1}}(k t) \\
& \geq \min \left\{H_{z_{n}, z_{n+1}}(t),{ }_{\delta} H_{z_{n}, \mathrm{QPR} z_{n}}(t),\right. \\
& { }_{\delta} H_{z_{n+1}, \mathrm{QPR} z_{n+1}}(t),{ }_{\delta} F_{R z_{n}, R z_{n+1}}(t), \\
& \left.{ }_{\delta} G_{P R z_{n}, P R z_{n+1}}(t)\right\}, \\
& \geq \min \left\{{ }_{\delta} H_{\mathrm{QPR} z_{n-1}, \mathrm{QPRz} z_{n}}(t)\right. \text {, } \\
& { }_{\delta} H_{\mathrm{QPR} z_{n}, \mathrm{QPR} z_{n+1}}(t) \text {, } \\
& { }_{\delta} F_{R Q P x_{n}, R Q P x_{n+1}}(t) \text {, }
\end{aligned}
$$

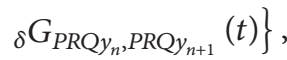

$$
\begin{aligned}
& \geq \min \left\{{ }_{\delta} H_{\mathrm{QPR} z_{n-1}, \mathrm{QPRz}}(t),\right. \\
& { }_{\delta} F_{R Q P x_{n-1}, R Q P x_{n}}(t) \text {, } \\
& \left.{ }_{\delta} G_{P R Q y_{n-1}, P R Q y_{n}}(t)\right\},
\end{aligned}
$$

on using inequalities (8) and (9). It follows easily by induction on using inequalities (8), (9), and (10) that

$$
\begin{aligned}
& F_{x_{n+1}, x_{n+2}}(t) \geq \min \left\{{ }_{\delta} F_{R Q P x_{1}, R Q P x_{2}}\left(\frac{t}{k^{n-1}}\right),\right. \\
& { }_{\delta} G_{P R Q y_{1}, P R Q y_{2}}\left(\frac{t}{k^{n-1}}\right), \\
& \left.{ }_{\delta} H_{\mathrm{QPR} z_{1}, \mathrm{QPRz} z_{2}}\left(\frac{t}{k^{n-1}}\right)\right\} \text {, } \\
& G_{y_{n+1}, y_{n+2}}(t) \geq \min \left\{{ }_{\delta} F_{R Q P x_{1}, R Q P x_{2}}\left(\frac{t}{k^{n-1}}\right)\right. \text {, } \\
& { }_{\delta} G_{P R Q y_{1}, P R Q y_{2}}\left(\frac{t}{k^{n-1}}\right),
\end{aligned}
$$

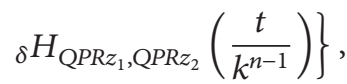

$$
\begin{aligned}
& H_{z_{n+1}, z_{n+2}}(t) \geq \min \left\{{ }_{\delta} F_{R Q P x_{1}, R Q P x_{2}}\left(\frac{t}{k^{n-1}}\right)\right. \text {, } \\
& { }_{\delta} G_{P R Q y_{1}, P R Q y_{2}}\left(\frac{t}{k^{n-1}}\right) \text {, } \\
& \left.{ }_{\delta} H_{\mathrm{QPRz} z_{1}, \mathrm{QPR} z_{2}}\left(\frac{t}{k^{n-1}}\right)\right\}
\end{aligned}
$$

for all $n=1,2, \ldots$. Since ${ }_{\delta} F_{R Q P x_{1}, R Q P x_{2}}\left(t / k^{n-1}\right) \rightarrow 1$, ${ }_{\delta} G_{P R Q y_{1}, P R Q y_{2}}\left(t / k^{n-1}\right) \rightarrow 1$, and ${ }_{\delta} H_{\mathrm{QPRz}}, \mathrm{QPRz_{2 }}\left(t / k^{n-1}\right) \rightarrow 1$ as $n \rightarrow \infty$, it follows that $\left\{x_{n}\right\},\left\{y_{n}\right\}$, and $\left\{z_{n}\right\}$ are Cauchy sequences in complete Menger space $X$ and so one has a limit $u$ in $X$. Similarly, the sequences $\left\{y_{n}\right\}$ and $\left\{z_{n}\right\}$ are also Cauchy sequences with limits $v$ and $w$ in the complete Menger spaces $Y$ and $Z$, respectively. Then we have

$$
\begin{aligned}
& \lim _{n \rightarrow \infty} R Q P x_{n}=\{u\}=\lim _{n \rightarrow \infty} R Q y_{n}, \\
& \lim _{n \rightarrow \infty} P R Q y_{n}=\{v\}=\lim _{n \rightarrow \infty} P R z_{n}, \\
& \lim _{n \rightarrow \infty} Q P R z_{n}=\{w\}=\lim _{n \rightarrow \infty} Q P x_{n} .
\end{aligned}
$$

Using the continuity of $P$ and $Q$, we have

$$
\begin{aligned}
& \lim _{n \rightarrow \infty} y_{n}=\lim _{n \rightarrow \infty} P x_{n}=P u=\{v\}, \\
& \lim _{n \rightarrow \infty} z_{n}=\lim _{n \rightarrow \infty} Q y_{n}=Q v=\{w\},
\end{aligned}
$$

and then we see that

$$
\mathrm{QPu}=\{w\} .
$$

Now we show that $u$ is a fixed point of $R Q P$. Applying inequality (5), we have

$$
\begin{gathered}
{ }_{\delta} F_{R Q P u, R Q P x_{n}}(k t) \geq{ }_{\delta} F_{R Q P u, R Q P x_{n-1}}(k t) \\
\geq \min \left\{F_{u, x_{n-1}}(t),{ }_{\delta} F_{u, R Q P u}(t),\right. \\
\delta F_{x_{n-1}, R Q P x_{n-1}}(t),{ }_{\delta} G_{P u, P x_{n-1}}(t), \\
\left.{ }_{\delta} H_{\mathrm{QPu}, \mathrm{QP} x_{n-1}}(t)\right\} .
\end{gathered}
$$


Since $P$ and $Q$ are continuous, it follows on letting $n \rightarrow \infty$ and using (12) that

$$
{ }_{\delta} F_{R Q P u, u}(k t) \geq{ }_{\delta} F_{u, R Q P u}(t) .
$$

Owing to Lemma $6, R Q P=\{u\}$ and so $u$ is a fixed point of $R Q P$. We now have

$$
R Q P u=R w=\{u\},
$$

on using (15) and then

$$
\begin{gathered}
P R Q v=P R Q P u=\{v\}, \\
Q P R w=Q P R Q v=Q v=\{w\},
\end{gathered}
$$

on using (14). Hence $v$ and $w$ are fixed points of PRQ and $Q P R$, respectively. Further, we see that

$$
R Q v=\{u\}, \quad P R w=\{v\} .
$$

To prove the uniqueness of $u$, suppose that $R Q P$ has another fixed point $u^{\prime}$. Then, using inequality (5), we have

$$
\begin{aligned}
&{ }_{\delta} F_{u^{\prime}, R Q P u^{\prime}}(k t) \geq{ }_{\delta} F_{R Q P u^{\prime}, R Q P u^{\prime}}(k t) \\
& \geq \min \left\{F_{u^{\prime}, u^{\prime}}(t),{ }_{\delta} F_{u^{\prime}, R Q P u^{\prime}}(t),\right. \\
&\left.\qquad{ }_{\delta} G_{P u^{\prime}, P u^{\prime}}(t),{ }_{\delta} H_{\mathrm{QPu} u^{\prime}, Q P u^{\prime}}(t)\right\} \\
& \geq \min \left\{{ }_{\delta} G_{P u^{\prime}, P u^{\prime}}(t),{ }_{\delta} H_{\mathrm{QPu} u^{\prime}, \mathrm{QP} u^{\prime}}(t)\right\} .
\end{aligned}
$$

Next, using inequality (6), we have

$$
\begin{aligned}
&{ }_{\delta} G_{P u^{\prime}, P u^{\prime}}(k t) \geq{ }_{\delta} G_{P R Q P u^{\prime}, P R Q P u^{\prime}}(k t) \\
& \geq \min \left\{G_{P u^{\prime}, P u^{\prime}}(t),{ }_{\delta} G_{P u^{\prime}, P R Q P u^{\prime}}(t),\right. \\
&\left.\qquad{ }_{\delta} H_{\mathrm{QPu}^{\prime}, \mathrm{QP} u^{\prime}}(t),{ }_{\delta} F_{R Q P u^{\prime}, R Q P u^{\prime}}(t)\right\} \\
& \geq \min \left\{{ }_{\delta} H_{\mathrm{QPu^{ \prime } , \mathrm { QPu }}}(t),{ }_{\delta} F_{R Q P u^{\prime}, R Q P u^{\prime}}(t)\right\},
\end{aligned}
$$

and using inequality (7), we obtain

$$
\begin{aligned}
& { }_{\delta} H_{\mathrm{QPu} u^{\prime}, \mathrm{QPu}}(k t) \geq{ }_{\delta} H_{\mathrm{QPRQP} u^{\prime}, \mathrm{QPRQPu}}(k t) \\
& \geq \min \left\{H_{\mathrm{QPu}^{\prime}, \mathrm{QP} u^{\prime}}(t),{ }_{\delta} H_{\mathrm{QP} u^{\prime}, \mathrm{QPRQPu} u^{\prime}}(t)\right. \text {, } \\
& { }_{\delta} F_{R Q P u^{\prime}, R Q P u^{\prime}}(t), \\
& \left.{ }_{\delta} G_{P R Q P u^{\prime}, P R Q P u^{\prime}}(t)\right\} \\
& \geq \min \left\{{ }_{\delta} F_{R Q P u^{\prime}, R Q P u^{\prime}}(t),\right. \\
& \left.{ }_{\delta} G_{P R Q P u^{\prime}, P R Q P u^{\prime}}(t)\right\} .
\end{aligned}
$$

It now follows easily from inequalities (22) and (23) that

$$
{ }_{\delta} G_{P R Q P u^{\prime}, P R Q P u^{\prime}}(t) \geq{ }_{\delta} F_{R Q P u^{\prime}, R Q P u^{\prime}}\left(\frac{t}{k}\right),
$$

and then

$$
{ }_{\delta} H_{\mathrm{QPu}, \mathrm{QP} u^{\prime}}(t) \geq{ }_{\delta} F_{R Q P u^{\prime}, R Q P u^{\prime}}\left(\frac{t}{k}\right) .
$$

Using inequalities (21), (24), and (25), we obtain

$$
\begin{aligned}
{ }_{\delta} F_{u^{\prime}, R Q P u^{\prime}}(t) & \geq{ }_{\delta} F_{R Q P u^{\prime}, R Q P u^{\prime}}(t) \\
& \geq{ }_{\delta} F_{R Q P u^{\prime}, R Q P u^{\prime}}\left(\frac{t}{k^{2}}\right) \\
& \geq \cdots{ }_{\delta} F_{R Q P u^{\prime}, R Q P u^{\prime}}\left(\frac{t}{k^{2 n}}\right),
\end{aligned}
$$

for all $n=1,2, \ldots$. Since ${ }_{\delta} F_{R Q P u^{\prime}, R Q P u^{\prime}}\left(t / k^{2 n}\right) \rightarrow 1$ as $n \rightarrow$ $\infty$, it follows that $R Q P u^{\prime}=u^{\prime}$ and so $R Q P u^{\prime}$ is a singleton. It then follows from inequality (25) that $Q P u^{\prime}$ is a singleton and from inequality (24) that $P u^{\prime}$ is a singleton. Using inequality (5) again, we have

$$
\begin{gathered}
F_{u, u^{\prime}}(k t)={ }_{\delta} F_{R Q P u, R Q P u^{\prime}}(k t) \\
\geq \min \left\{F_{u, u^{\prime}}(t), F_{u, u}(t), F_{u^{\prime}, R Q P u^{\prime}}(t),\right. \\
\left.G_{P u, P u^{\prime}}(t), H_{\mathrm{QPu}, \mathrm{QP} u^{\prime}}(t)\right\} \\
\geq \min \left\{G_{P u, P u^{\prime}}(t), H_{\mathrm{QPu}, \mathrm{QP} u^{\prime}}(t)\right\} .
\end{gathered}
$$

Next using inequality (6), we have

$$
\begin{gathered}
G_{P u, P u^{\prime}}(k t)={ }_{\delta} G_{P R Q P u, P R Q P u^{\prime}}(k t) \\
\geq \min \left\{G_{P u, P u^{\prime}}(t), G_{P u, P R Q P u}(t),\right. \\
G_{P u^{\prime}, P R Q P u^{\prime}}(t), H_{\mathrm{QPu}, \mathrm{QP} u^{\prime}}(t), \\
\left.F_{R Q P u, R Q P u^{\prime}}(t)\right\} \\
=\min \left\{H_{\mathrm{QPu}, \mathrm{QPu} u^{\prime}}(t), F_{u, u^{\prime}}(t)\right\},
\end{gathered}
$$

and using inequality (7), we have

$$
\begin{gathered}
H_{\mathrm{QPu}, \mathrm{QPu} u^{\prime}}(k t) \geq{ }_{\delta} H_{\mathrm{QPRQPu}, \mathrm{QPRQPu}}(k t) \\
\geq \min \left\{H_{\mathrm{QPu}, \mathrm{QPu} u^{\prime}}(t), H_{\mathrm{QPu}, \mathrm{QPRQPu}}(t),\right. \\
H_{\mathrm{QPu^{ \prime } , \mathrm { QPRQPu }}}(t), F_{R Q P u, R Q P u^{\prime}}(t), \\
\left.G_{P R Q P u, P R Q P u^{\prime}}(t)\right\} \\
=\min \left\{F_{u, u^{\prime}}(t), G_{P u, P u^{\prime}}(t)\right\} .
\end{gathered}
$$

It follows from inequalities (27), (28), and (29) that $F_{u, u^{\prime}}(t)=$ 1 , which shows the uniqueness of $u$. The uniqueness of $v$ and $w$ can be verified similarly.

Example 9. Consider $X=[0,2], Y=[1,5]$, and $Z=[0,10]$ and define

$$
\begin{aligned}
F_{x, y}(t) & =G_{x, y}(t)=H_{x, y}(t) \\
& = \begin{cases}\frac{t}{t+|x-y|}, & \text { if } t>0 ; \\
0, & \text { if } t=0,\end{cases}
\end{aligned}
$$


for all $x, y \in X$. Then $(X, \mathscr{F}, \Delta),(Y, \mathscr{G}, \Delta)$, and $(Z, H, \Delta)$ are three complete Menger spaces with continuous $t$-norm $\Delta(a, b)=\min \{a, b\}$ for all $a, b \in[0,1]$. Define the set-valued mappings $P: X \rightarrow \mathscr{C} \mathscr{B}(Y), Q: Y \rightarrow \mathscr{C} \mathscr{B}(Z)$ and $R: Z \rightarrow \mathscr{C} \mathscr{B}(X)$ by $P x=[1+x, 2]$ for all $x \in X$, $Q y=[2 y+1,5]$ for all $y \in Y$ and

$$
R z= \begin{cases}{\left[\frac{z}{5}, 1\right],} & \text { if } z \in[0,5] \\ \left(1, \frac{z}{5}\right], & \text { if } z \in(5,10] .\end{cases}
$$

We can easily verify the inequalities with $k=1 / 2 \in(0,1)$. Thus all the conditions of Theorem 8 are satisfied and $R Q P$ has a unique fixed point $\{1\}$ in $\mathscr{C} \mathscr{B}(X), P R Q$ has a unique fixed point $\{2\}$ in $\mathscr{C} \mathscr{B}(Y)$, and $Q P R$ has a unique fixed point $\{5\}$ in $\mathscr{C} \mathscr{B}(Z)$. Hence, $P(1)=\{2\}, Q(2)=\{5\}$, and $R(5)=\{1\}$, and so $R Q P(1)=R Q(2)=R(5)=\{1\}, P R Q(2)=P R(5)=$ $P(1)=\{2\}$, and $Q P R(5)=Q P(1)=Q(2)=\{5\}$.

Corollary 10. Let $(X, \mathscr{F}, \Delta),(Y, \mathscr{G}, \Delta)$ and $(Z, H, \Delta)$ be three complete Menger spaces, where $\Delta$ is a continuoust-norm. If $P$ is a continuous mapping of $X$ into $Y, Q$ is a continuous mapping of $Y$ into $Z$ and $R$ is a mapping of $Z$ into $X$ satisfying the inequalities

$$
\begin{gathered}
F_{R Q P x, R Q P x^{\prime}}(k t) \geq \min \left\{F_{x, x^{\prime}}(t), F_{x, R Q P x}(t),\right. \\
F_{x^{\prime}, R Q P x^{\prime}}(t), G_{P x, P x^{\prime}}(t), \\
\left.H_{Q P x, \mathrm{QPx^{ \prime }}}(t)\right\} \\
G_{P R Q y, P R Q y^{\prime}}(k t) \geq \min \left\{G_{y, y^{\prime}}(t), G_{y, P R Q y}(t),\right. \\
G_{y^{\prime}, P R Q y^{\prime}}(t), H_{\mathrm{Q} y, \mathrm{Q} y^{\prime}}(t), \\
\left.F_{R Q y, R Q y^{\prime}}(t)\right\} \\
H_{\mathrm{QPRz}, \mathrm{QPRz^{ \prime }}}(k t) \geq \min \left\{H_{z, z^{\prime}}(t), H_{z, \mathrm{QPR} z}(t),\right. \\
H_{z^{\prime}, \mathrm{QPR} z^{\prime}}(t), F_{R z, R z^{\prime}}(t), \\
\left.G_{P R z, P R z^{\prime}}(t)\right\}
\end{gathered}
$$

for all $x, x^{\prime} \in X, y, y^{\prime} \in Y, z, z^{\prime} \in Z, k \in(0,1)$, and $t>0$. Then $R Q P$ has a unique fixed point $u$ in $X, P R Q$ has a unique fixed point $v$ in $Y$ and $Q P R$ has a unique fixed point $w$ in $Z$. Further, $P u=v, Q v=w$ and $R w=u$.

Example 11. Let $X=[0,2], Y=[1,3], Z=[3,5]$ and $F_{x, y}(t)$, $G_{x, y}(t)$ and $H_{x, y}(t)$ be defined as Example 9. Then $(X, \mathscr{F}, \Delta)$, $(Y, \mathscr{G}, \Delta)$, and $(Z, H, \Delta)$ be three complete Menger spaces with continuous $t$-norm $\Delta(a, b)=\min \{a, b\}$ for all $a, b \in[0,1]$. Define the mappings $P: X \rightarrow Y, Q: Y \rightarrow Z$ and $R: Z \rightarrow$ $X$ by $P x=x+1$ for all $x \in X, Q y=y+2$ for all $y \in Y$ and

$$
R z= \begin{cases}\frac{z}{4}, & \text { if } z \in[3,4] ; \\ \frac{z+1}{3}, & \text { if } z \in(4,5] .\end{cases}
$$

Also the inequalities (32) can be verified with $k=1 / 2 \in(0,1)$. Thus all the conditions of Corollary 10 are satisfied and $R Q P$ has a unique fixed point 1 in $X, P R Q$ has a unique fixed point 2 in $Y$, and $Q P R$ has a unique fixed point 4 in $Z$. Further, $P(1)=$ 2, $Q(2)=4$, and $R(4)=1$; that is, $R Q P(1)=R Q(2)=R(4)=$ $1, P R Q(2)=P R(4)=P(1)=2$, and $Q P R(4)=Q P(1)=$ $Q(2)=4$.

The following corollaries are the natural results due to Theorem 8 and Corollary 10.

Corollary 12. Let $(X, \mathscr{F}, \Delta)$ and $(Y, \mathscr{G}, \Delta)$ be two complete Menger spaces, where $\Delta$ is a continuous t-norm. If $P$ is a continuous mapping of $X$ into $\mathscr{C} \mathscr{B}(Y), Q$ is a mapping of $Y$ into $\mathscr{C} \mathscr{B}(X)$ satisfying the inequalities

$$
\begin{gathered}
{ }_{\delta} F_{\mathrm{QPx}, \mathrm{QPX} x^{\prime}}(k t) \geq \min \left\{F_{x, x^{\prime}}(t),{ }_{\delta} F_{x, \mathrm{Q} P x}(t),\right. \\
\left.{ }_{\delta} F_{x^{\prime}, \mathrm{Q} P x^{\prime}}(t),{ }_{\delta} G_{P x, P x^{\prime}}(t)\right\} \\
{ }_{\delta} G_{P Q y, P Q y^{\prime}}(k t) \geq \min \left\{G_{y, y^{\prime}}(t),{ }_{\delta} G_{y, P Q y}(t),\right. \\
\left.{ }_{\delta} G_{y^{\prime}, P Q y^{\prime}}(t),{ }_{\delta} F_{\mathrm{Q} y, \mathrm{Q} y^{\prime}}(t)\right\}
\end{gathered}
$$

for all $x, x^{\prime} \in X, y, y^{\prime} \in Y, k \in(0,1)$, and $t>0$. Then $Q P$ has a unique fixed point $u$ in $X$ and $P Q$ has a unique fixed point $v$ in $Y$.

Corollary 13. Let $(X, \mathscr{F}, \Delta)$ and $(Y, \mathscr{G}, \Delta)$ be two complete Menger spaces, where $\Delta$ is a continuous t-norm. If $P$ is a continuous mapping of $X$ into $Y, Q$ is a mapping of $Y$ into $X$ satisfying the inequalities

$$
\begin{gathered}
F_{\mathrm{QPx}, \mathrm{QP} x^{\prime}}(k t) \geq \min \left\{F_{x, x^{\prime}}(t), F_{x, \mathrm{QPx}}(t),\right. \\
\left.F_{x^{\prime}, \mathrm{Q} P x^{\prime}}(t), G_{P x, P x^{\prime}}(t)\right\}, \\
G_{P Q y, P Q y^{\prime}}(k t) \geq \min \left\{G_{y, y^{\prime}}(t), G_{y, P Q y}(t),\right. \\
\left.G_{y^{\prime}, P Q y^{\prime}}(t), F_{\mathrm{Q} y, \mathrm{Q} y^{\prime}}(t)\right\}
\end{gathered}
$$

for all $x, x^{\prime} \in X, y, y^{\prime} \in Y, k \in(0,1)$, and $t>0$. Then $Q P$ has a unique fixed point $u$ in $X$ and $P Q$ has a unique fixed point $v$ in $Y$.

Remark 14. Theorem 8 generalizes the result of Jain et al. [6] and Fisher [2]. Corollaries 10-13 extend the results of Fisher and Türkoğlu [3, Theorem 1, Corollary 1] to PM-spaces.

\section{Acknowledgment}

The authors are thankful to anonymous referees for their valuable suggestions and comments to improve the paper.

\section{References}

[1] B. Fisher, "Fixed points on two metric spaces," Glasnik Matematički, vol. 16, no. 36, pp. 333-337, 1981.

[2] B. Fisher, "Related fixed points on two metric spaces," Kobe University, vol. 10, no. 1, pp. 17-26, 1982. 
[3] B. Fisher and D. Türkoğlu, "Related fixed points for set valued mappings on two metric spaces," International Journal of Mathematics and Mathematical Sciences, vol. 23, no. 3, pp. 205-210, 2000.

[4] V. K. Chourasia and B. Fisher, "Related fixed points for two pairs of set valued mappings on two metric spaces," Hacettepe Journal of Mathematics and Statistics, vol. 32, pp. 27-32, 2003.

[5] S. Jain and B. Fisher, "A related fixed point theorem for three metric spaces," Hacettepe Journal of Mathematics and Statistics, vol. 31, pp. 19-24, 2002.

[6] R. K. Jain, H. K. Sahu, and B. Fisher, "Related fixed point theorems for three metric spaces," Novi Sad Journal of Mathematics, vol. 26, no. 1, pp. 11-17, 1996.

[7] R. K. Namdeo, S. Jain, and B. Fisher, "A related fixed point theorem for two pairs of mappings on two complete metric spaces," Hacettepe Journal of Mathematics and Statistics, vol. 32, pp. 7-11, 2003.

[8] M. Telci, "Fixed points on two complete and compact metric spaces," Applied Mathematics and Mechanics, vol. 22, no. 5, pp. 564-568, 2001.

[9] B. D. Pant, "Relation between fixed points in Menger spaces," Journal of the Indian Academy of Mathematics, vol. 24, no. 1, pp. 135-142, 2002.

[10] B. D. Pant and S. Kumar, "A related fixed point theorem for two pairs of mappings in two Menger space," Varähmihir Journal of Mathematical Sciences, vol. 6, no. 2, pp. 471-476, 2006.

[11] B. Schweizer and A. Sklar, Probabilistic Metric Spaces, NorthHolland Series in Probability and Applied Mathematics, NorthHolland Publishing Co., New York, NY, USA, 1983.

[12] K. Menger, "Statistical metrics," Proceedings of the National Academy of Sciences of the United States of America, vol. 28, pp. 535-537, 1942.

[13] S. N. Mishra, "Common fixed points of compatible mappings in PM-spaces," Mathematica Japonica, vol. 36, no. 2, pp. 283-289, 1991.

[14] S.-S. Chang, Y. J. Cho, and S. M. Kang, Nonlinear Operator Theory in Probabilistic Metric Spaces, Nova Science Publishers Inc., Huntington, NY, USA, 2001. 


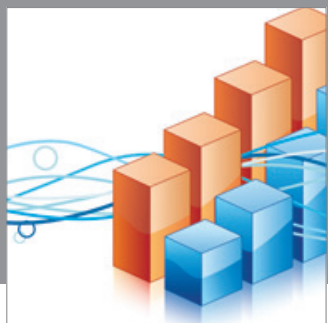

Advances in

Operations Research

mansans

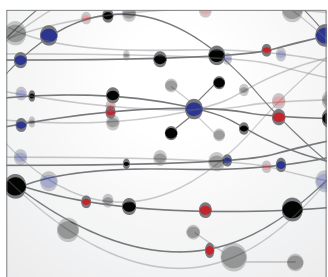

The Scientific World Journal
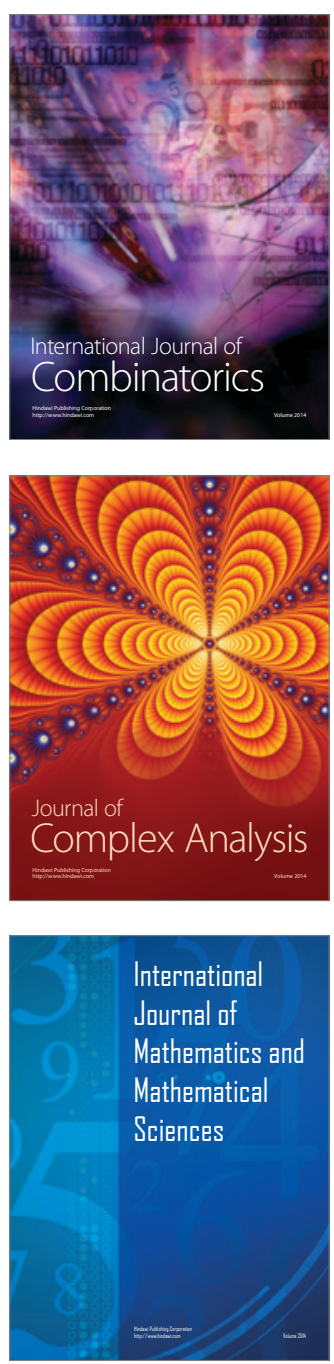
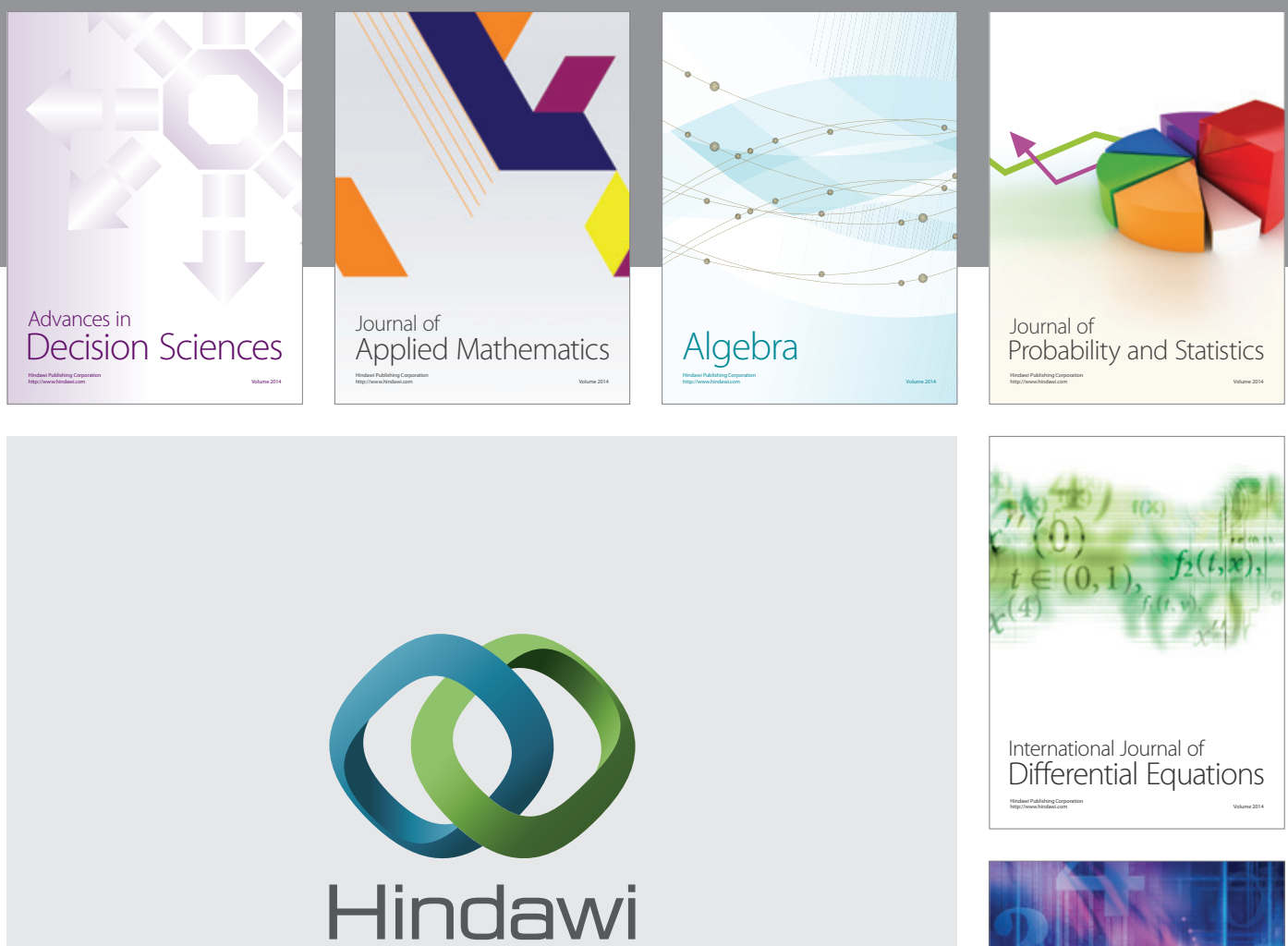

Submit your manuscripts at http://www.hindawi.com
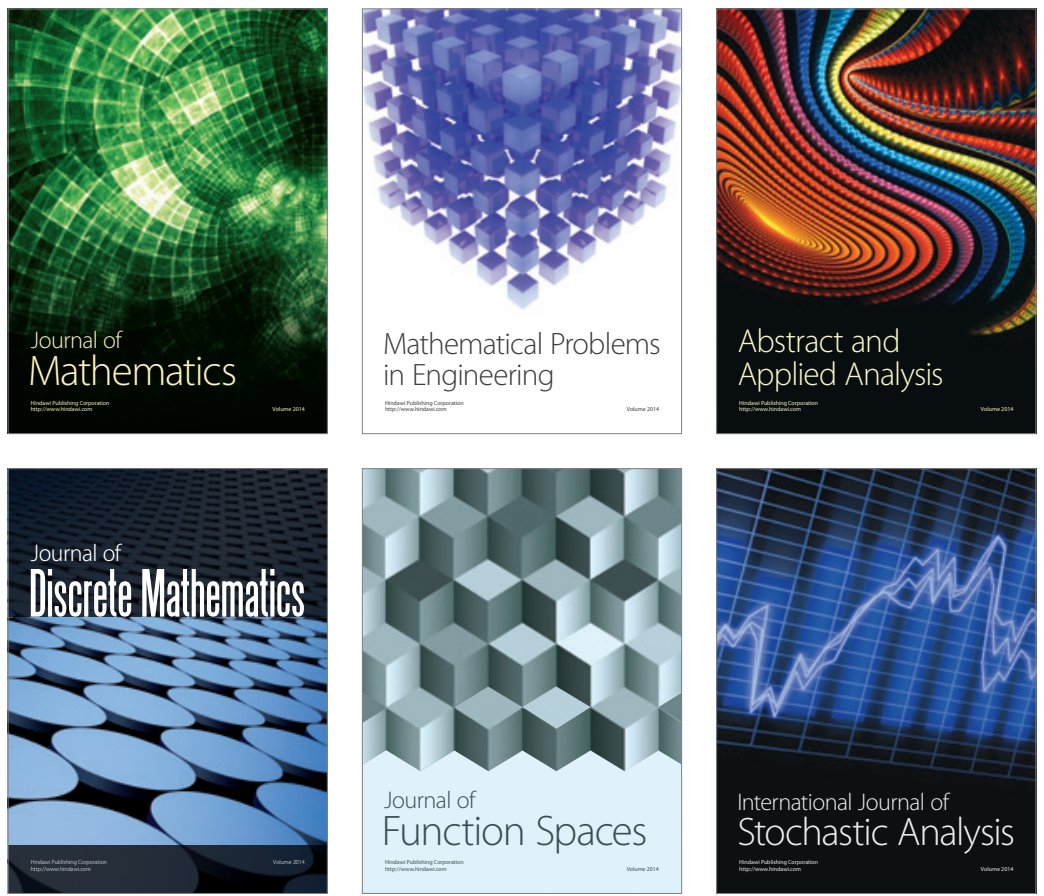

Journal of

Function Spaces

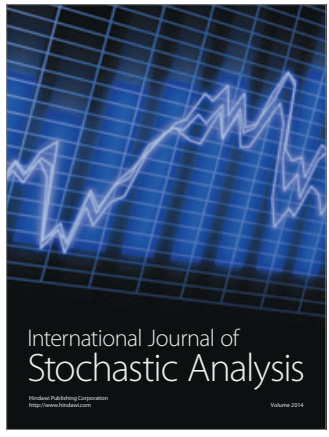

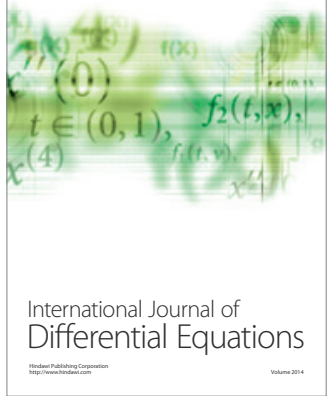
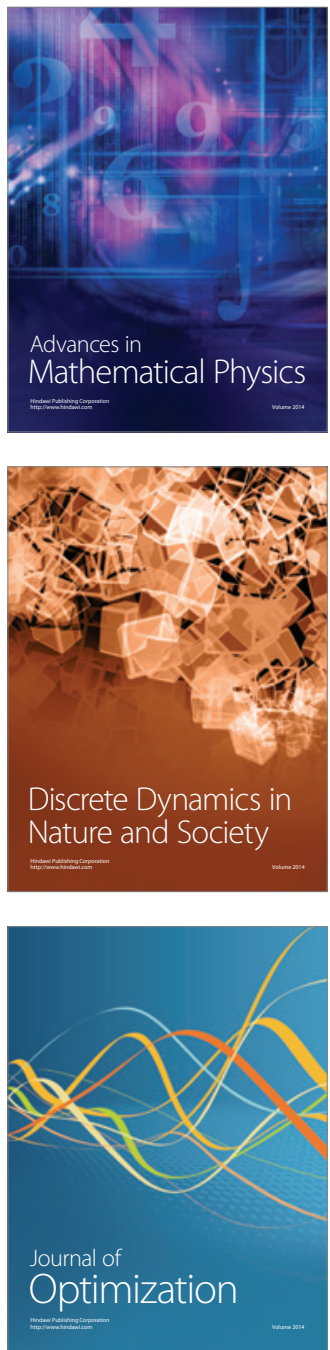